\title{
On the universality of void density profiles
}

\author{
E. Ricciardelli ${ }^{1}$, V. Quilis ${ }^{1,2}$ and J. Varela ${ }^{3}$ \\ ${ }^{1}$ Departament d'Astronomia i Astrofisica, Universitat de Valencia, c/ Dr. Moliner 50, E-46100 \\ - Burjassot, València, Spain \\ email: elena.ricciardelli@gmail.com \\ ${ }^{2}$ Centro de Estudios de Física del Cosmos de Aragón (CEFCA), Plaza San Juan 1, 44001 \\ Teruel, Spain \\ ${ }^{3}$ Observatori Astronòmic, Universitat de València, E-46980 Paterna, València, Spain
}

\begin{abstract}
The massive exploitation of cosmic voids for precision cosmology in the upcoming dark energy experiments, requires a robust understanding of their internal structure, particularly of their density profile. We show that the void density profile is insensitive to the void radius both in a catalogue of observed voids and in voids from a large cosmological simulation. However, the observed and simulated voids display remarkably different profile shapes, with the former having much steeper profiles than the latter. We ascribe such difference to the dependence of the observed profiles on the galaxy sample used to trace the matter distribution. Samples including low-mass galaxies lead to shallower profiles with respect to the samples where only massive galaxies are used, as faint galaxies live closer to the void centre. We argue that galaxies are biased tracers when used to probe the matter distribution within voids.
\end{abstract}

Keywords. cosmology: large-scale structure of universe, cosmology: observations, methods: numerical

\section{Introduction}

Cosmic voids are recently attracting growing interest thanks to their potential in probing cosmological parameters. In particular, voids are the ideal candidate for probing the expansion history of the Universe through the Alcock-Paczynski test, using the average shape of stacked voids (e.g., Lavaux \& Wandelt 2012, Sutter et al. 2012). The huge potentiality of voids for precision cosmology requires a robust knowledge of their internal structure, particularly of the density profiles. Recent works have studied the void density profiles without reaching a consensus on the functional form that can reproduce such profiles and on their dependence on void size (e.g., Ricciardelli et al. 2013, Ricciardelli et al. 2014, Hamaus et al. 2014, Nadathur et al. 2014). In the present work, we study the density profiles of voids in a catalogue of observed voids and in voids from a large cosmological simulation and assess their universality, that we intend as their insensitivity on void sizes. In doing so, we also provide a robust determination of the systematic effects arising when using different mass tracers.

The catalogue of observed voids is drawn from SDSS-DR7 with the same method adopted by Varela et al. 2012. Voids are identified as empty spheres, devoid of galaxies brighter than $M_{r}-5 \operatorname{logh}=-20.17$. For the present analysis, only voids with radius larger $7 h^{-1} M p c$ are considered, resulting in 4453 voids. As for the simulations, we use a version of the MASCLET code (Quilis 2004), that has been designed to follow the formation and evolution of low density regions, to simulate a box of comoving size length $512 h^{-1} M p c$. In the simulation, we identify more than 3000 voids larger than $7 h^{-1} M p c$. 


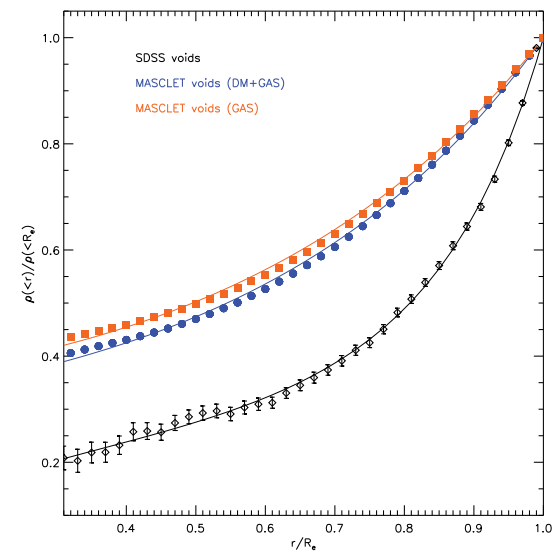

Figure 1. Stacked density profiles for all voids larger than $7 h^{-1} M p c$ identified in the SDSS database (black diamonds) and the best-fit model (black line). The coloured symbols indicate the stacked profiles of the simulated voids larger than $7 h^{-1} M p c$, computed using the total density field (blue circles) and the gas density field (orange squares). The solid coloured lines indicate the best-fits for each curve.

\section{Results}

In Fig. 1 we show the comparison between simulated and observed stacked profiles. The simulated profiles are computed using the density field to trace the matter distribution, whereas in the observed voids the density is traced by the luminous galaxies. The stacked profile is fitted by the two-parameters law proposed in Ricciardelli et al. (2013):

$$
\frac{\rho(<r)}{\rho_{e}}=\left(\frac{r}{R_{e}}\right)^{\alpha} \exp \left[\left(\frac{r}{R_{e}}\right)^{\beta}-1\right]
$$

where $\rho(<r)$ is the density enclosed within the void-centric distance $r, \rho_{e}$ is the density enclosed within the void effective radius $R_{e}$ and $\alpha$ and $\beta$ are the best-fit parameters to be obtained from the fit. The functional form expressed in Eq. 2.1 turns out to be adequate in reproducing with good accuracy both the observed and simulated profiles, although the free parameters used in the two cases are significantly different, as the observed profile appears much steeper than the simulated one.

A possible reason for the steepness of the observed density profile could lie in the different tracers used to measure the void density profiles. To assess the impact of the mass tracers, we have built volume limited samples of galaxies up to a given redshift and complete down to the corresponding threshold mass limit (see Ricciardelli et al. 2014). It is worth to emphasize that the choice of the galaxy sample used only affects the recovered density profiles, leaving the sample of voids unchanged. In Fig. 2 we show the recovered density profiles using the different galaxy samples. The profiles appear to steepen as galaxies at higher redshift and higher stellar mass are used. Interestingly, the profiles traced with the faintest galaxy samples approach the simulated profiles shown in Fig. 1. The steepening is particularly evident in the evolution of $\alpha$, that becomes progressively higher as more massive galaxies are concerned. On the other hand, $\beta$ does not show any clear dependence on the tracers, as the $\beta$ beta values are just scattered around the reference values. We argue that the steepness of the observed profile, with respect to the simulated ones, can be explained by the absence of tracers in the innermost regions of the observed voids. It is not clear however whether such absence could be solved by using deeper data or it is just a consequence of the galaxy bias. We notice that hat a similar comparison of density profiles measured with different samples of galaxies has 


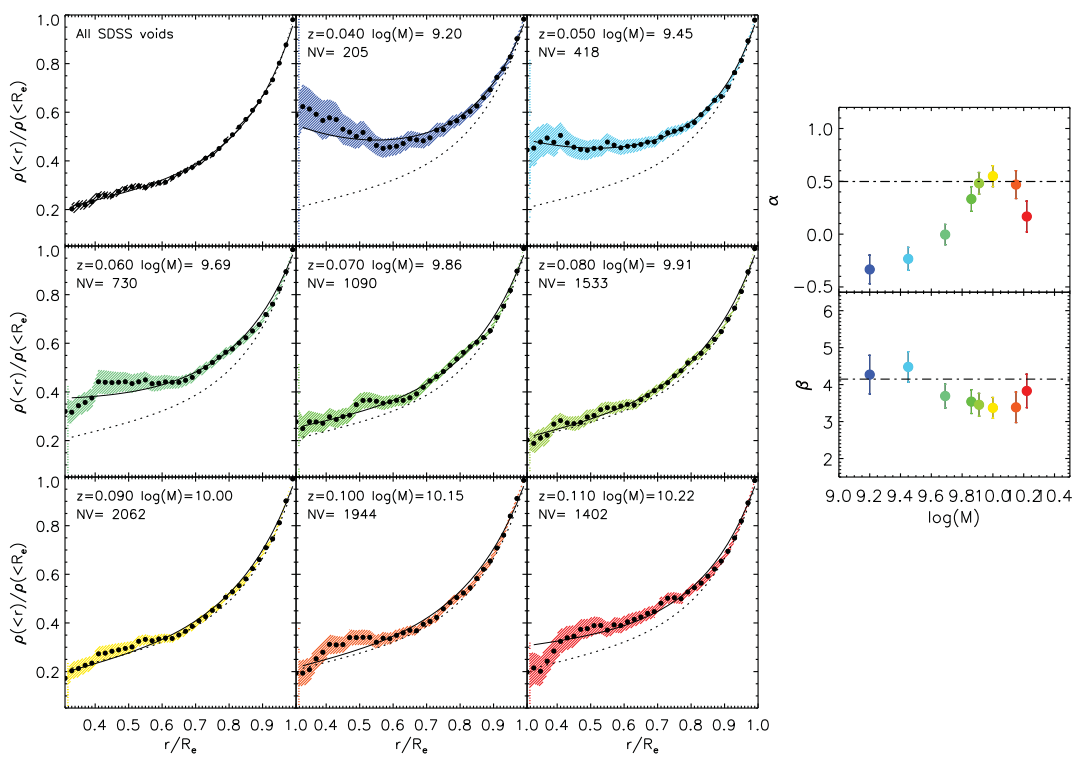

Figure 2. Dependence of the observational void density profiles on the choice of the density tracers. Different panels show the density profiles for samples of voids lying at redshift below that indicated and using, as mass tracers, the galaxies more massive than the threshold mass at that redshift. The black points indicate the radial value of the stacked void, colored shaded regions show the confidence regions determined by means of a bootstrapping, and the black solid line is the best fit. The dotted black line reported in all the panels is the best-fit density profiles of the stack drawn from the parent sample (first panel). The dependence of the best-fit parameters on the threshold mass of the galaxies used is shown in the smaller panels on the right.

been shown by Nadathur et al. $(2013,2014)$, but they do not find any dependence of the profile on the magnitude of the tracers. However, their galaxy samples are relatively bright $(M r<-18.16+5 \log (h))$. Void galaxies in our SDSS catalogue, are, by definition, fainter than $M r=-20.17+5 \log (h)$, hence allowing us to probe the profiles using also galaxies with very low mass. In fact, if only the highest mass bins were concerned, we would not observe such dependence of the profile on the galaxy mass. Furthermore, we refer to mass density, whereas the above authors only consider number densities.

To assess the dependence of the void density profiles on the void radius, we refer to the observed voids and divide the void sample in equi-populated subsamples, having $\sim 300$ voids each. The profiles for different void radii are shown in Fig. 3. All the best-fit parameters, $\alpha$ and $\beta$, fall within 1-2 $\sigma$ of the reference values, derived by fitting the profile of the parent sample at $z<0.08$, without any dependence on the radius. Indeed, the best-fit profile derived for the parent sample (dotted line) is compatible with the profile shape in all the size bins. Such insensitivity of the void density profile on the void radius is obtained also when using the simulated voids (Ricciardelli et al. 2013, Ricciardelli et al. 2014).

\section{Conclusions}

We have shown that the void density profiles recovered by means of the observed and simulated voids share the same qualitative shape, showing a significant underdensity in the centre and a sharp density increase approaching the void edges. Both profiles can be well described by the functional form proposed in Ricciardelli et al. (2013). Such profile, 


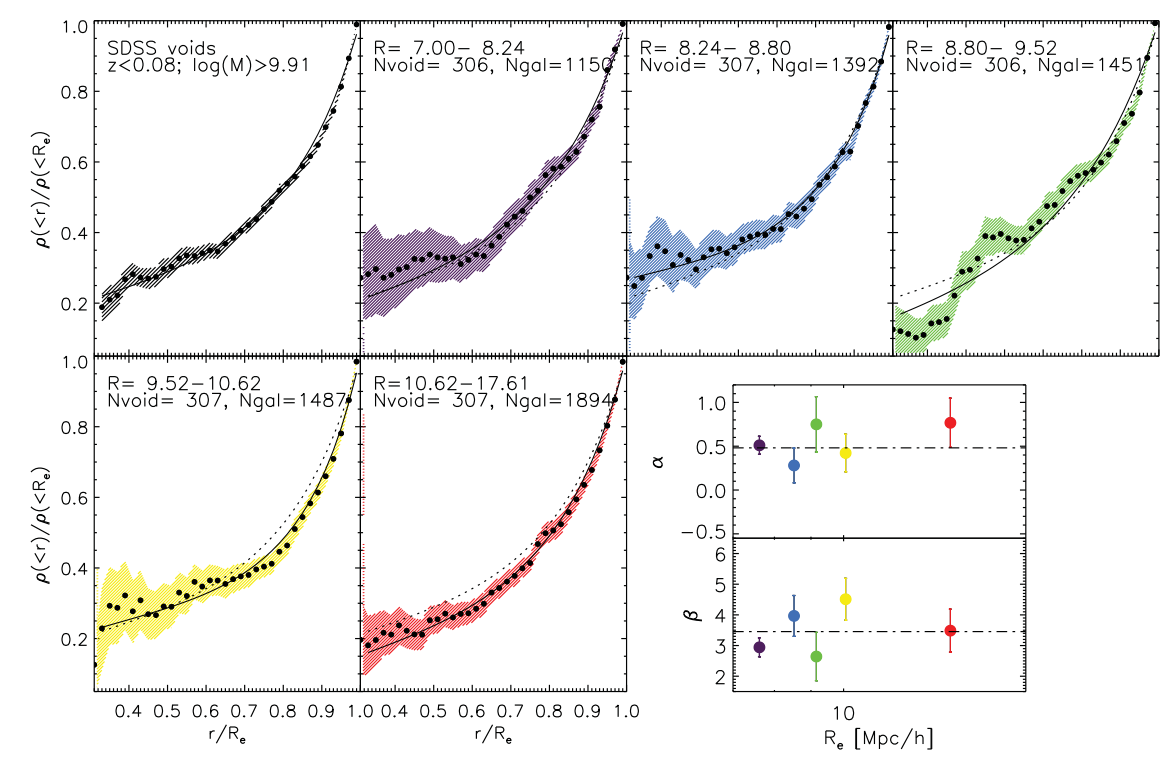

Figure 3. Observational void density profiles as a function of void radius. The first panel shows the density profile measured from an homogenous sample of galaxies, with voids lying at $z<0.08$ and the mass tracers having stellar mass above $10^{9.9} M_{\odot}$. In the other panels, we show the density profiles of voids within different size bins. The black points indicate the radial value of the stacked void, colored shaded regions show the confidence regions determined by means of a bootstrapping, and the black solid line is the best fit. The dotted black line reported in all the panels is the best-fit density profile shown in the first panel. The lower-right panels show the dependence of the best-fit parameters $\alpha$ and $\beta$ on void radius.

if properly rescaled, does not depend on the void size, hence we refer to it as universal density profile. It nevertheless has a strong dependence on the mass of the tracers used to constrain the profiles. Within the observed voids, the density profiles recovered by means of faint samples of galaxies are shallower than those determined through the brighter galaxies. The reason for that lies in the galaxy mass segregation within voids. In fact, faint galaxies are those living closer to the void centre and, thus, allow to probe the matter distribution even in the innermost part of the voids.

\section{References}

Hamaus, N., Sutter, P. M., \& Wandelt, B. D., 2014, PhRvL, 112, 251302

Lavaux, G. \& Wandelt, B. D., 2012, ApJ, 754, 109

Quilis, V., 2004, MNRAS, 352, 1426

Nadathur, S. \& Hotchkiss, S., 2013, arXiv, arXiv:1310.2791

Nadathur, S., Hotchkiss, S., Diego, J. M., Iliev, I. T., Gottlöber, S., Watson, W. A., \& Yepes, G., 2014, arXiv, arXiv:1407.1295

Ricciardelli, E., Quilis, V., \& Planelles, S., 2013, MNRAS, 434, 1192

Ricciardelli, E., Quilis, V., \& Varela, J., 2014, MNRAS, 440, 601

Sutter, P. M., Lavaux, G., Wandelt, B. D., \& Weinberg, D. H., 2012, ApJ, 761, 187

Varela, J., Betancort-Rijo, J., Trujillo, I., \& Ricciardelli, E., 2012, ApJ, 744, 82 